\title{
Utility of endometrial sampling prior to risk-reducing hysterectomy in a patient with Lynch syndrome
}

\author{
Melissa K Frey', Gizelka David-West ${ }^{1}$, Khushbakhat R Mittal2 ${ }^{2}$, Franco M Muggia ${ }^{3}$ and Bhavana Pothuri' \\ 1Department of Obstetrics and Gynecology, Division of Gynecologic Oncology, New York University Langone Medical Center, New York, NY 10016, USA \\ ${ }^{2}$ Department of Pathology, New York University Langone Medical Center, New York, NY 10016, USA \\ ${ }^{3}$ Department of Medicine, New York University Langone Medical Center, New York, NY 10016, USA
}

Correspondence to: Melissa K Frey. Email: melissa.frey@nyumc.org

\begin{abstract}
Occult endometrial cancer is occasionally discovered in women with Lynch syndrome undergoing risk-reducing hysterectomy. The case presented here demonstrates that preoperative endometrial sampling can help detect these occult cancers; however, there are currently no recommendations for this preoperative intervention. A 50-year-old woman with Lynch syndrome underwent endometrial sampling prior to planned risk-reducing hysterectomy and bilateral salpingo-oophorectomy. The endometrial biopsy demonstrated a serous endometrial cancer. The patient was counselled regarding the diagnosis and revised operative plan, which now included staging, prior to surgery. Although the prevalence of occult endometrial cancer at the time of risk-reducing surgery in women with Lynch syndrome remains unknown, preoperative endometrial sampling may allow for improved patient counselling and surgical planning in this population, and can help avoid a subsequent surgery for staging.
\end{abstract}

Keywords: colorectal neoplasms, hereditary nonpolyposis, endometrial neoplasms, hysterectomy, risk reduction surgery, uterine neoplasms

Published: $18 / 01 / 2016$

Received: 07/09/2015

ecancer 2016, 10:613 DOI: 10.3332/ecancer.2016.613

Copyright: $\odot$ the authors; licensee ecancermedicalscience. This is an Open Access article distributed under the terms of the Creative Commons Attribution License (http://creativecommons.org/licenses/by/3.0), which permits unrestricted use, distribution, and reproduction in any medium, provided the original work is properly cited. 


\section{Introduction}

Women with Lynch syndrome have an autosomal dominant mutation in one of the DNA mismatch repair genes resulting in an increased lifetime risk for colorectal and endometrial cancer in addition to cancers of the ovary, stomach, hepatobiliary tract, pancreas, small bowel, urinary tract, and central nervous system. The current National Comprehensive Cancer Network (NCCN) guidelines recommend that individuals with Lynch syndrome undergo colorectal cancer screening with colonoscopy [1]. There is less data to support gynaecologic screening. However, because of high lifetime risk of endometrial cancer in women with Lynch syndrome the NCCN and other consensus guidelines recommend annual or biennial endometrial sampling beginning at age 30-35 years and risk-reducing hysterectomy and bilateral salpingo-oophorectomy in women who have completed childbearing [1, 2]. The role of endometrial sampling preceding risk-reducing surgery is yet to be addressed. We present a case of an asymptomatic patient found on preoperative endometrial sampling to have serous endometrial cancer, raising the question of whether preoperative endometrial sampling should become the standard of care for Lynch patients undergoing risk-reducing hysterectomy.

\section{Case presentation}

A 50-year-old, gravida zero, Ashkenazi Jewish woman underwent screening for Lynch syndrome. Her paternal grandfather was diagnosed with colon cancer at age 80, paternal uncle diagnosed with colon cancer at age 50, and two paternal second cousins diagnosed with premenopausal endometrial cancer. Both the patient and her father were found to have the Lynch syndrome mutation MSH6 3959del4, which results in premature truncation of the MSH6 protein at amino acid position 1325 [3]. Following this screening result, the patient was immediately referred for risk-reducing gynaecologic surgery.

The patient had no personal history of cancer or gynaecologic pathology and reported regular menses with a 20 -year history of oral contraceptive use. The patient underwent a pelvic ultrasound which demonstrated an $8 \mathrm{~mm}$ endometrial echo with heterogeneity, likely representing small benign endometrial polyps. She was referred to a gynaecologic oncologist who counselled her regarding the option for risk-reducing surgery, which would include total hysterectomy and bilateral salpingo-oophorectomy. The gynaecologic oncologist performed an endometrial biopsy in the office after explaining to the patient that despite normal menses and a lack of gynaecologic symptoms, a preoperative diagnosis of endometrial cancer would affect the surgical plan and therefore a preoperative endometrial biopsy could provide valuable information. The endometrial biopsy demonstrated endometrial serous adenocarcinoma (Figure 1). The patient underwent a preoperative CT scan which showed no evidence of metastatic disease. She was counselled that based on the preoperative pathologic diagnosis, the recommended surgery now included pelvic and para-aortic lymph node dissection, omentectomy, and peritoneal biopsies.

The patient underwent an uncomplicated laparoscopic total hysterectomy, bilateral salpingo-oophorectomy, pelvic and para-aortic lymph node dissection, omentectomy, and peritoneal biopsies. The final pathology demonstrated a stage IA uterine papillary serous cancer with no myometrial invasion. The patient received three cycles of chemotherapy with carboplatin and paclitaxel with no evidence of disease at the conclusion of treatment. She will continue screening for other Lynch-associated cancers.
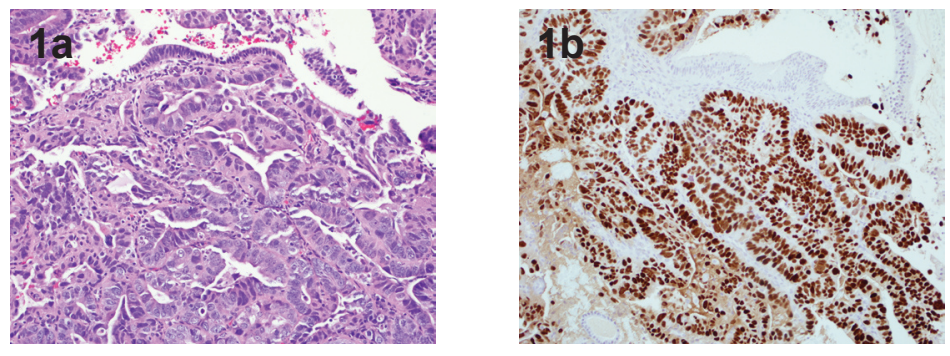

Figure 1. 1a. Glandular variant of uterine serous carcinoma. Notice the high grade nuclei, H \& E 20x. 1b. Immunostain for p53 highlights all tumor nuclei. Benign epithelium and stroma are not stained. p53 immunostain 20x. 


\section{Discussion}

Women with Lynch syndrome undergoing risk-reducing hysterectomy are at risk for having an occult endometrial cancer, however the magnitude of risk remains unknown. The estimated cumulative lifetime risk for endometrial cancer in Lynch syndrome varies by report and mutation, ranging from $21-71 \%$. The MSH6 3959del4 mutation (as in the current case) is associated with the greatest risk of endometrial cancer, affecting $71 \%$ of women by age 70 [3]. Prior case reports have described occult endometrial cancer discovered in patients with Lynch syndrome undergoing risk-reducing surgery [4, 5]. Lachiewicz et al [6] recently published the first study to explore the prevalence of occult gynaecologic malignancy at the time of risk-reducing surgery in patients with Lynch syndrome. Four of 24 (16.7\%) patients in this study were noted to have cancer on final pathology, three with endometrial cancers (12.5\%), and one with ovarian cancer (4.2\%).

Currently, there are no recommendations in place for endometrial sampling prior to risk reducing surgery and, in fact, gynaecologic cancer surveillance in Lynch syndrome patients remains an area of debate. Two studies have reported a lack of efficacy of screening transvaginal ultrasounds in women with Lynch syndrome [7, 8]. More recently, however, Lécuru et al [9] found that ultrasonography alone was effective for diagnosing endometrial cancer with $100 \%$ sensitivity and $100 \%$ negative predictive value. In the case presented here, the ultrasound report findings showed what appeared to be benign endometrial polyps. The results of studies evaluating screening endometrial sampling have been similarly conflicting $[8,10]$. Women with Lynch syndrome have a high lifetime risk of endometrial cancer and many will develop the disease prior to menopause when irregular bleeding may not be recognised as an early symptom [11]. As a result, despite conflicting results of screening efficacy, the NCCN, American Congress of Obstetricians and Gynaecologists, and other consensus groups recommend endometrial cancer surveillance as an option in this high-risk population [1, 2].

The reported cases of occult endometrial cancer discovered at the time of risk-reducing hysterectomy pose the question of whether endometrial sampling should be a routine component of the preoperative evaluation. Our case is another example of an occult endometrial cancer but additionally it highlights the clinical benefit of obtaining information on a cancer diagnosis prior to surgery. The patient described in this case was diagnosed with a serous endometrial cancer prior to surgical intervention. Patients with Lynch syndrome can have either type I (endometrioid) or type II (high-risk histologic subtypes) endometrial cancers. Broaddus et al [12] performed a pathologic evaluation of 50 Lynch-associated endometrial cancers and found that $14 \%$ were type II. Furthermore, serous endometrial cancers in this population present at an earlier age than in the general population (46 years versus 65-68 years), as it occurred with our patient [12, 13]. Risk-reducing hysterectomy and bilateral salpingo-oophorectomy is an incomplete surgery for serous endometrial cancers and therefore patients with occult disease diagnosed only on final surgical pathology will require a second surgery. Because of the preoperative diagnosis, our patient was able to receive thorough preoperative counselling about the high-risk pathology and surgical plan, and she underwent complete surgical staging in a single procedure, which likely would not have been possible without the preoperative diagnosis of endometrial cancer. This patient was already being followed by a gynaecologic oncologist but many patients undergoing risk-reducing surgery will have their surgery performed by general gynaecologic surgeons. For these patients, a positive preoperative biopsy will allow for early referral to subspecialty care.

\section{Conclusion}

Further prospective studies are required to determine the true prevalence of occult endometrial cancers at the time of risk-reducing surgery. However, in the meantime, surgeons may want to consider preoperative endometrial sampling in women with Lynch syndrome undergoing risk-reducing surgery, especially if in a small cohort the incidence was as high as $12.5 \%$. As in this case, the diagnosis of cancer enabled a pre-operative discussion with the patient, and this in turn helped to avoid a second surgery for staging.

\section{Conflicts of interest}

There are no conflicts of interest to report.

\section{Acknowledgments}

There are no acknowledgements. 


\section{Ethics and dissemination}

The patient provided written consent for the publication of this case report.

\section{References}

1. NCCN Guidelines Version 2 (2014) High-risk colorectal cancer syndromes

2. Committee on Practice Bulletins-Gynecology and Society of Gynecologic Oncology (2014) ACOG Practice Bulletin No. 147: Lynch syndrome Obstet Gynecol 124 1042-54 DOI: 10.1097/01.AOG.0000456325.50739.72 PMID: 25437740

3. Hendriks YM et al (2004) Cancer risk in hereditary nonpolyposis colorectal cancer due to MSH6 mutations: impact on counseling and surveillance Gastroenterology 127(1) 17-25 DOI: 10.1053/j.gastro.2004.03.068 PMID: 15236168

4. Pistorius SR et al (2001) Combined molecular and clinical approach for decision making for surgery in HNPCC patients: a report on three cases in two families Int J Colorectal Dis 16(6) 402-7 DOI: 10.1007/s003840100349

5. Chung L et al (2003) Unexpected endometrial cancer at prophylactic hysterectomy in a woman with hereditary nonpolyposis colon cancer Obstet Gynecol 102(5 Pt 2) 1152-5 DOI: 10.1016/S0029-7844(03)00699-9

6. Lachiewicz MP et al (2014) Prevalence of occult gynecologic malignancy at the time of risk reducing and nonprophylactic surgery in patients with Lynch syndrome Gynecol Oncol 132(2) 434-7 DOI: 10.1016/j.ygyno.2013.10.033

7. Dove-Edwin I et al (2002) The outcome of endometrial carcinoma surveillance by ultrasound scan in women at risk of hereditary nonpolyposis colorectal carcinoma and familial colorectal carcinoma Cancer 94(6) 1708-12 DOI: 10.1002/cncr.10380 PMID: 11920532

8. Renkonen-Sinisalo L et al (2007) Surveillance for endometrial cancer in hereditary nonpolyposis colorectal cancer syndrome Int J Cancer 120(4) 821-4 DOI: 10.1002/ijc.22446

9. Lecuru F et al (2010) Contribution of ultrasonography to endometrial cancer screening in patients with hereditary nonpolyposis colorectal cancer/Lynch syndrome Int J Gynecol Cancer 20(4) 583-7 DOI: 10.1111/IGC.0b013e3181d7283a PMID: 20686377

10. Helder-Woolderink JM et al (2013) The additional value of endometrial sampling in the early detection of endometrial cancer in women with Lynch syndrome Gynecol Oncol 131(2) 304-8 DOI: 10.1016/j.ygyno.2013.05.032 PMID: 23769810

11. Lindor NM et al (2006) Recommendations for the care of individuals with an inherited predisposition to Lynch syndrome: a systematic review JAMA 296(12) 1507-17 DOI: 10.1001/jama.296.12.1507 PMID: 17003399

12. Broaddus RR et al (2006) Pathologic features of endometrial carcinoma associated with HNPCC: a comparison with sporadic endometrial carcinoma Cancer 106(1) 87-94 DOI: $10.1002 / \mathrm{cncr} .21560$

13. Christopherson WM, Alberhasky RC and Connelly PJ (1982) Carcinoma of the endometrium. II. Papillary adenocarcinoma: a clinical pathological study, 46 cases Am J Clinic Pathol 77(5) 534-40 